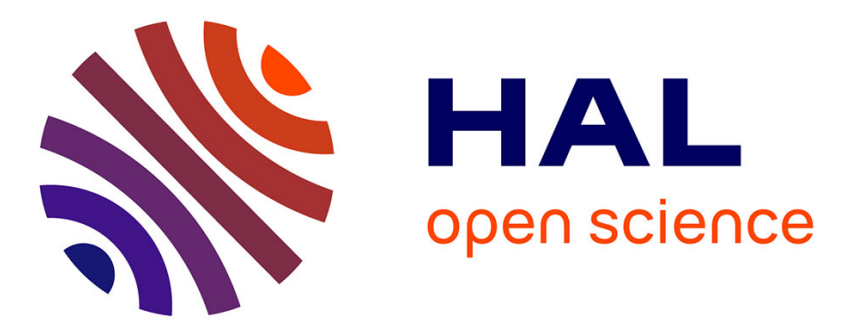

\title{
Multistrange baryon production in heavy ion reactions at the SPS
}

\author{
F. Antinori, W. Beusch, I.J. Bloodworth, R. Caliandro, N. Carrer, D. Di Bari, \\ S. Di Liberto, D. Elia, D. Evans, K. Fanebust, et al.
}

\section{- To cite this version:}

F. Antinori, W. Beusch, I.J. Bloodworth, R. Caliandro, N. Carrer, et al.. Multistrange baryon production in heavy ion reactions at the SPS. Nuclear Physics A, 2001, 685, pp.407-413. in2p3-00019669

\section{HAL Id: in2p3-00019669 https://hal.in2p3.fr/in2p3-00019669}

Submitted on 5 Sep 2001

HAL is a multi-disciplinary open access archive for the deposit and dissemination of scientific research documents, whether they are published or not. The documents may come from teaching and research institutions in France or abroad, or from public or private research centers.
L'archive ouverte pluridisciplinaire HAL, est destinée au dépôt et à la diffusion de documents scientifiques de niveau recherche, publiés ou non, émanant des établissements d'enseignement et de recherche français ou étrangers, des laboratoires publics ou privés. 

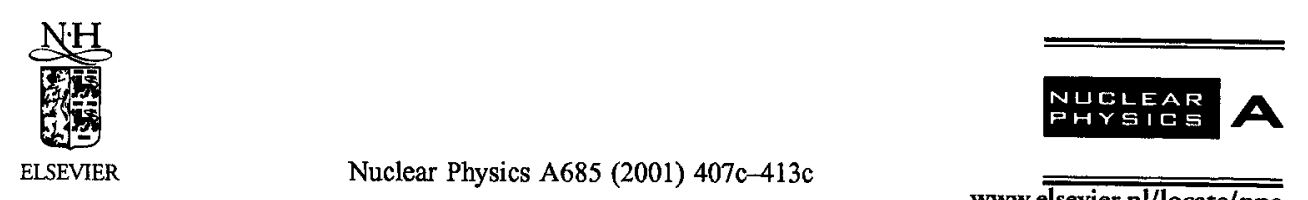

www.e elsevier.nl/locate/npe

Multistrange baryon production in heavy ion reactions at the SPS

Presented by H.Helstrup ${ }^{3}$ for the WA97 Collaboration:

F. Antinori ${ }^{6,10}$, W. Beusch ${ }^{6}$, I.J. Bloodworth ${ }^{4}$, R. Caliandro ${ }^{1}$, N. Carrer ${ }^{6}$, D. Di Bari ${ }^{1}$,

S. Di Liberto ${ }^{12}$, D. Elia ${ }^{1}$, D. Evans ${ }^{4}$, K. Fanebust ${ }^{2}$, F. Fayazzadeh ${ }^{9}$, R.A. Fini ${ }^{1}$,

J. Ftáčnik ${ }^{7}$, B. Ghidini ${ }^{1}$, G. Grella ${ }^{12}$, M. Gulino ${ }^{5}$, H. Helstrup ${ }^{3}$, M. Henriquez ${ }^{9}$, A.K. Holme ${ }^{9}$, D. Huss ${ }^{8}$, A. Jacholkowski ${ }^{1}$, G.T. Jones ${ }^{4}$, J.B. Kinson ${ }^{4}$, K. Knudson ${ }^{6}$, I. Králik ${ }^{7}$, V. Lenti ${ }^{1}$, R. Lietava ${ }^{6}$, R.A. Loconsole ${ }^{1}$, G. Løvhøiden ${ }^{9}$, V. Manzari ${ }^{1}$, M.A. Mazzoni ${ }^{12}$, F. Meddi ${ }^{12}$, A. Michalon ${ }^{14}$, M.E. Michalon-Mentzer ${ }^{14}$, M. Morando $^{10}$, P.I. Norman ${ }^{4}$, B. Pastirčák ${ }^{7}$, E. Quercigh ${ }^{6}$, D. Röhrich ${ }^{2}$, G. Romano ${ }^{13}$, K. Šafařík ${ }^{6}$, L. Sándor ${ }^{6,7}$, G. Segato ${ }^{10}$, P. Staroba ${ }^{11}$, M. Thompson ${ }^{4}$, G.D. Torrieri ${ }^{4}$, J. Urbán ${ }^{7}$, T. Vik ${ }^{9}$ O. Villalobos Baillie ${ }^{4}$, T. Virgili ${ }^{13}$, M.F. Votruba ${ }^{4}$ and P. Závada ${ }^{11}$.

${ }^{1}$ Dipartimento I.A. di Fisica dell'Università e del Politecnico di Bari and Sezione INFN, Bari, Italy

${ }^{2}$ Fysisk institutt, Universitetet i Bergen, Bergen, Norway

3 Høgskolen i Bergen, Bergen, Norway

${ }^{4}$ School of Physics and Astronomy, University of Birmingham, Birmingham, UK

${ }^{5}$ University of Catania and INFN, Catania, Italy

${ }^{6}$ CERN, European Laboratory for Particle Physics, Geneva, Switzerland

${ }^{7}$ Institute of Experimental Physics, Slovak Academy of Sciences, Košice, Slovakia

${ }^{8}$ GRPHE, Université de Haute Alsace, Mulhouse, France

${ }^{9}$ Fysisk institutt, Universitetet i Oslo, Oslo, Norway .

10 Dipartimento di Fisica dell'Università and Sezione INFN, Padua, Italy

11 Institute of Physics, Academy of Sciences of the Czech Republic, Prague, Czech Republic

12 Dipartimento di Fisica dell'Università "La Sapienza" and Sezione INFN, Rome,Italy

13 Dipartimento di Scienze Fisiche "E.R. Caianiello" dell'Università and INFN, Salerno, Italy

${ }^{14}$ Institut de Recherches Subatomiques, IN2P3/ULP, Strasbourg, France

Strange particle production in proton-beryllium, proton-lead and lead-lead interactions as measured by the WA97 experiment at the CERN SPS is presented. Strangeness enhancement is observed to increase with the strangeness content of the particle, reaching a factor 15 for $\Omega$ s. Inverse slope systematics are shown, including data from other experiments.

\section{Introduction}

An enhanced production of strange particles has been put forward as one of the main signals for the identification of a phase transition from hadronic matter to a quark gluon plasma (QGP) [1,2]. 


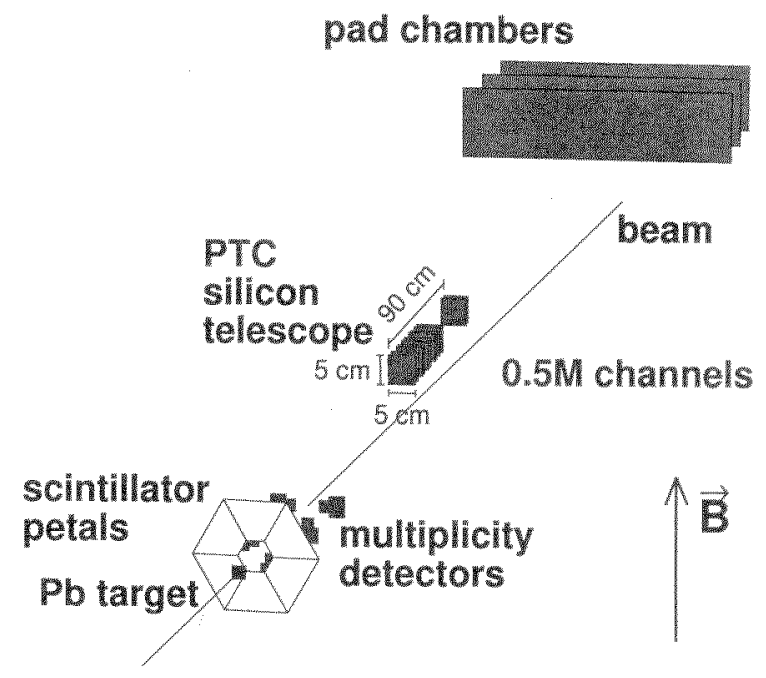

Figure 1. WA97 experimental setup

In particular, multistrange particles and antiparticles are expected to provide a sensitive observable to identify quark matter formation [3] since, in a QGP scenario, the enhancement is expected to increase with the strangeness content of the particle (statistical hadronization). In a purely hadronic scenario (i.e. no QGP) this is not to be expected, since multistrange hadron production is hindered with respect to singly strange production by high tresholds and low cross sections.

The WA97 experiment has measured mid-rapidity production yields of strange and multistrange baryons and antibaryons carrying strangeness $(0,1,2$ and 3$)$ in lead-lead, proton-lead and proton-beryllium interactions. The enhancements observed by WA97, and their increase with the strangeness content of the particle, form one of the main motivations for the announcement of the observation of a new state of matter observed at CERN [4].

\section{Experimental setup}

The WA97 setup, outlined in figure 1 , is discussed in more detail in refs [5,6]. Tracks are identified in a silicon telescope containing 7 pixel planes with $75 \times 500 \mu^{2}$ pixel size, and 10 microstrip planes with a $50 \mu \mathrm{m}$ pitch. Tracks are identified using the first compact part of the telescope, where 6 pixel planes and 5 microstrip planes are placed close together over a distance of $30 \mathrm{~cm}$. The remaining silicon planes, as well as MWPCs with pad cathode readout, are used to improve the momentum resolution.

Event multiplicities are measured by fast scintillators to provide a trigger signal, which 


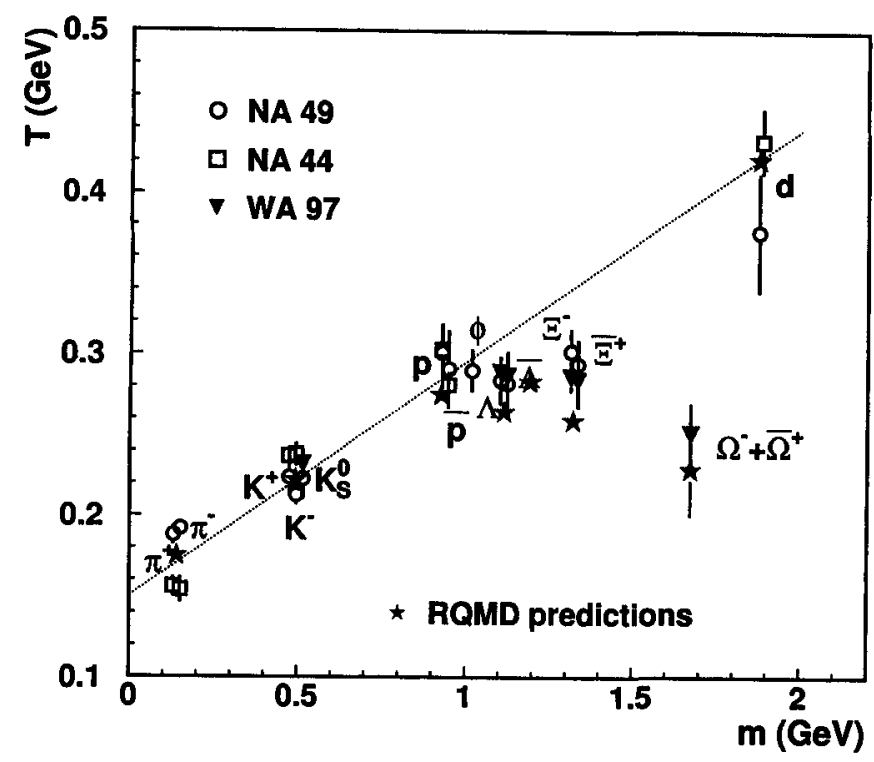

Figure 2. Dependence of the $m_{\mathrm{T}}$ spectra inverse slopes $T$ on the particle mass $\mathrm{m}$.

selects the $40 \%$ most central events in lead-lead interactions. Detailed multiplicity measurements are made by two arrays of silicon microstrips.

In proton-induced reactions, additional scintillators were used in the trigger to select events where at least a minimum number of particles (one or two) pass through the telescope.

Hyperons are identified by their decay patterns. All decays are required to take place before the first plane of the telescope, and all decay tracks are required to pass through the compact part of the telescope. As no experimental particle identification of decay tracks is provided, $\Omega$ s kinematically ambiguous with the $\Xi$ mass hypothesis are explicitly excluded from the analysis.

Further details of the reconstruction procedure are discussed in refs $[6,7]$.

\section{Transverse mass spectra}

Inverse slope parameters $T$ have been extracted by fitting the transverse mass spectra by the formula

$\frac{1}{m_{T}} \frac{d N}{d m_{T}} \propto \exp \left(-\frac{m_{T}}{T}\right)$

A compilation of inverse slope parameters from NA44, NA49 and WA97 is shown in figure 2[8]. The line represents an approximation of the RQMD model calculation for 


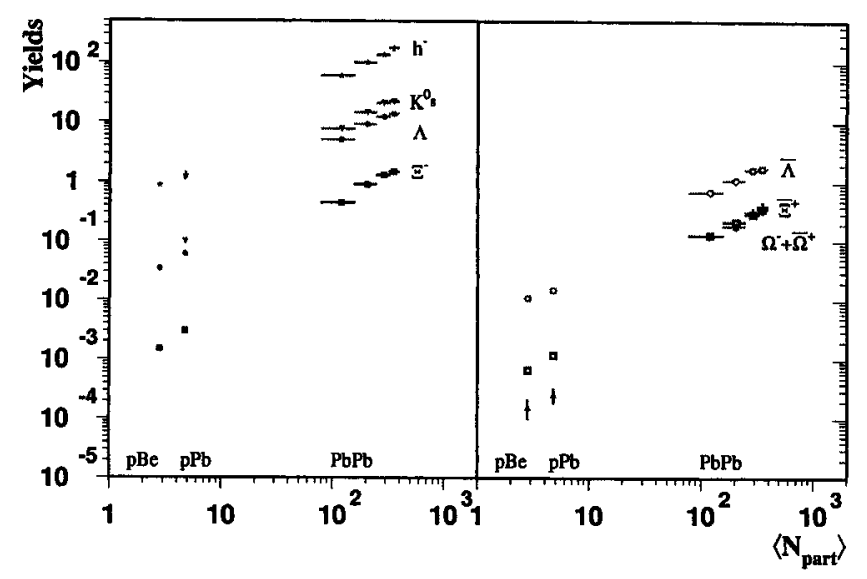

Figure 3. Yields per unit of rapidity at central rapidity as a function of number of wounded nucleons.

non-strange particles [9]. The inverse slopes increases with increasing particle mass, with exception of $\Omega$ s and possibly $\Xi s$. This suggests that $\Omega$ s decouple from the interaction zone at an earlier stage than the other particles, due to their small hadronic cross sections [8].

Figure 2 also show values obtained from RQMD calculations [9]. The calculated values agree well with the experimentally observed ones, also for $\Omega$ s.

\section{Centrality measurement}

The centrality measurement is based on the charged particle multiplicity measured by the multiplicity microstrips. The lead-lead data sample is divided into four multiplicity classes, with approximately equal numbers of measured hyperons in each multiplicity class. The average number of participants for each multiplicity class is determined by a Wounded Nucleon Model [10] fit. The procedure to obtain the number of wounded nucleons in each multiplicity class is described in detail in ref. [11].

The number of wounded nucleons in proton-induced reactions has been determined as an average value for inelastic collisions in the framework of the Glauber model [12].

\section{Particle yields}

The particle yields per unit of rapidity at central rapidity is presented for particles carrying $0,1,2$ and 3 levels of strangeness in figure 3 . Particles with at least one quark in common with the nucleon are shown in the left part of the figure, particles with no quarks in common with the incoming nucleons are shown to the right.

The particle ratios calculated from these yields, along with measurements by other 


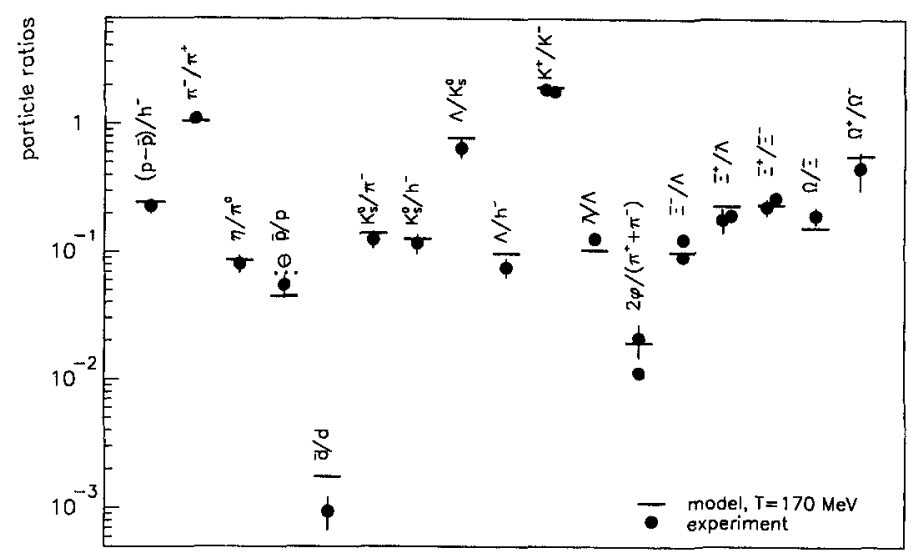

Figure 4. Thermal model fit to particle yields in lead-lead interactions [13].

experiments, have been compared with thermal model predictions in ref. [13]. A wide range of particle ratios can all be fitted with a common temperature parameter of 170 $\mathrm{MeV}$, as is shown in figure 4 .

In figure 5 the yields are normalised to the value observed in proton-beryllium interactions and divided by the corresponding (average) number of participants in the interactions, displaying the enhancement factor for each particle species compared to protonberyllium interactions on the vertical scale. The particles are grouped the same way as discussed for figure 3.

No enhancement beyond proportionality with the number of participants is observed when proton-lead data are compared to proton-beryllium data.

Within the measured centrality sample in lead-lead interactions, corresponding to the $40 \%$ most central events, the enhancement factors do not depend on centrality. All yields per participant measured in lead-lead interactions are significantly higher than the ones observed in proton-induced reactions. These results call for a closer investigation of the intermediate centrality region, to search for a possible transition behaviour.

Even negative particles (mostly pions) show a small enhancement in lead-lead interactions. This could be interpreted by rescattering effects in a purely hadron scenario, or by entropy production in a QGP case.

As no significant centrality dependence of the enhancement factors within the lead sample is observed, average enhancement factors over all multiplicity classes and for each of the particle species presented are shown in figure 6. Within each group, the enhancement 


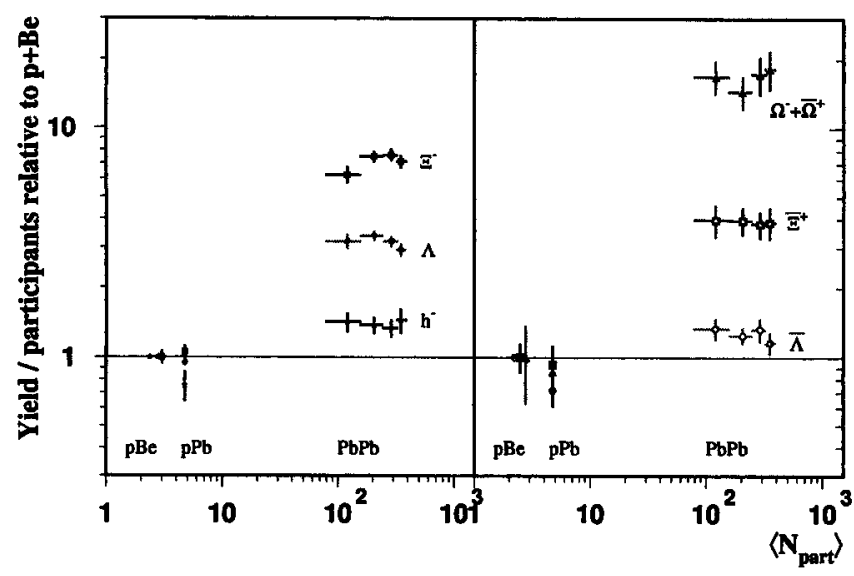

Figure 5. Yields per unit of rapidity at central rapidity scaled to pBe yields and divided by number of participants.

factor increases with the strangeness content of the particle, reaching a factor 15 for $\left(\Omega^{-}+\right.$ $\bar{\Omega}^{+}$). This enhancement pattern is not explained by currently used hadronic models. Collecting all multiplicity classes allows separate presentation of $\Omega^{-}$and $\bar{\Omega}^{+}$. As expected, their enhancement factors are very similar.

\section{Outlook}

The experimental programme of WA97 has been taken over by the NA57 experiment. The aim of this experiment is to extend the centrality range by measuring more peripheral lead-lead interactions at $160 \mathrm{GeV}$ per nucleon and by using a lower beam energy $(40 \mathrm{GeV}$ per nucleon).

\section{Conclusions}

The WA97 experiment has measured particle spectra for particles carrying all levels of strangeness. Strangeness enhancement is observed when yields measured in lead-lead interactions are compared to yields measured in proton-induced reactions. A thermal model with a common temperature of $170 \mathrm{MeV}$ has shown good agreement with a wide range of particle ratios measured by several experiments. The enhancement factor increases with the strangeness content of the particle, reaching a factor 15 for $\Omega$. This enhancement pattern cannot be explained by standard hadronic models. It provides strong evidence for the production of deconfined matter in central $\mathrm{Pb}-\mathrm{Pb}$ collisions at SPS energies. 


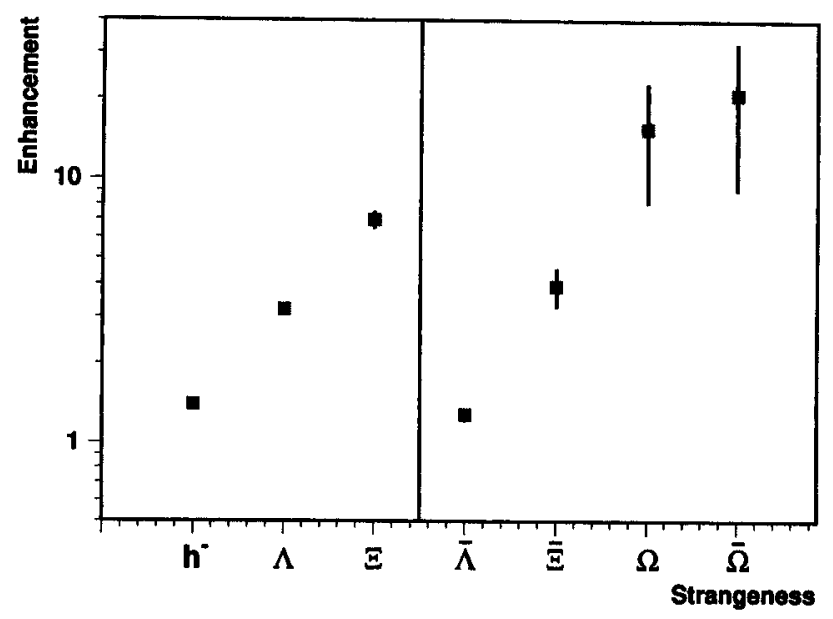

Figure 6. Enhancement factors for each particle species. The enhancement factors are obtained from collecting data from all multiplicity classes.

\section{REFERENCES}

1. J. Rafelski, Phys. Rep. 88(1982) 331

2. P. Koch, B. Müller and J. Rafelski, Phys. Rep. 142(1986) 167

3. J. Rafelski, Phys. Lett. B262(1991)333.

4. U. Heinz and M. Jacob, nucl-th/0002042

5. F. Antinori et al. (WA97 Collaboration), Nucl. Phys. A590(1995) 139c

6. E. Andersen et al. (WA97 Collaboration), Phys. Lett. B433(1998) 209

7. E. Andersen et al. (WA97 Collaboration), Phys. Lett. B449(1999) 401

8. F. Antinori et al. (WA97 Collaboration), Eur.Phys.J. C14(2000)633.

9. H. van Hecke, H. Sorge and N. Xu, Phys.Rev.Lett. 81(1998)5764.

10. A. Białas, M. Bleszyński and W. Czyż, Nucl. Phys. B111(1976) 461

11. F. Antinori, et al. (WA97 and NA57 Collaborations), to be published in Eur.Phys.J. C.

12. C. Y. Wong, Introduction to High-Energy Heavy-Ion Collisions, World Scientific, Singapore, 1994, p. 251-264.

13. P. Braun-Munzinger, I. Heppe and J. Stachel, Phys. Lett. B465(1999)15. 